\title{
Project Documentation for Android
}

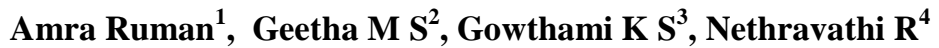 \\ Abstract: This application is designed with the aim to provide an easier way of referencing various projects, technical papers, \\ articles, technical articles and journals related to the current technical trend thus helping the students in their technical documentation \\ activities.
}

Keywords: Referencing, documentation, articles, journals, papers, projects

\section{INTRODUCTION}

Project documentation plays a major role in any technical work. Project documentation provides an easy way of understanding the entire design and working of the project for both technical and non-technical people. In this project we provide various works done by the technical people to make it useful for the students and also giving them the platform to upload their own projects or papers module by module as and when they develop project that are based on their interest. It also improves one's skill on writing technical papers and journals. We develop an application on desktop for android. Android is popular with technology companies that require a ready-made, low-cost and customizable operating system for high-tech devices. Its open nature has encouraged a large community of developers and enthusiasts to use the open-source code as a foundation for community-driven projects, which deliver updates to older devices, add new features for advanced users or bring Android to devices originally shipped with other operating systems.

\section{RELATED WORK}

Automated user documentation for android:

Modern computing involve a larger integration of mobile devices most of them running on the android platform. Developing for android is facilitated by the android SDK provided by Google and is based on the model of apps distributed typically through the play store. The value of most android app is limited by the quality of the user documentation that is provided with the app. There is no standardized way to document the app and no best practice, guidance is offered by Google for the help system implementation inside the app. The purpose of this is to investigate the most effective and productive way to document an android app.

Wiki-side Page Structure:

The page structure conventions have the purpose of facilitating easy extraction of relevant info from the wiki, and automated building of the help pages for every screen in the application.

Apptivo:

Project management's primary challenge is to achieve all of project goals and objectives. The primary constraints of Projects App are scope, budget, quality, invoicing and time tracking. You can plan, collaborate with teams, cost effective, fix the rate for Project, can disclose your documents, and exchange views among the team.

\section{Methodology}

Here we use the concept of classification algorithm of data mining. Data mining is a process of extraction of useful information and patterns from huge data. It is also called as knowledge discovery process, knowledge mining from data, knowledge extraction or data pattern analysis [1]. The Classification is the one of the major role in Data mining. Basically classification is a 2-step process; the first step is supervised learning for the sake of the predefined class label for training data set. Second step is classification accuracy evaluation. Data mining is a logical process that is used to search through large amount of data in order to find useful data.

Classification is the task of generalizing known structure to apply to new data. For example, an email program might attempt to classify an email as legitimate or spam. Common algorithms include

- Decision Tree

- K-Nearest Neighbor

- Support Vector Machines

- Naive Bayesian Classification 
- Neural Networks.

A Decision Tree Classifier consists of a decision tree generated on the basis of instances. A decision tree is a classifier expressed as a recursive partition of the instance space.

K-Nearest neighbor classifiers are based on learning by analogy. The training samples are described by $\mathrm{n}$ dimensional numeric attributes. Each sample represents a point in an n-dimensional space. In this way, all of the training samples are stored in an $\mathrm{n}$-dimensional pattern space.

SVM was first introduced by Vapnik [2] and has been very effective method for regression, classification and general pattern recognition. It is considered a good classifier because of its high generalization performance without the need to add a priori knowledge, even when the dimension of the input space is very high.

A Bayesian network (BN) consists of a directed, acyclic graph and a probability distribution for each node in that graph given its immediate predecessors [3]. A Bayes Network Classifier is based on a Bayesian network which represents a joint probability distribution over a set of categorical attributes. It consists of two parts, the directed acyclic graph $\mathrm{G}$ consisting of nodes and arcs and the conditional probability tables.

An artificial neural network (ANN), often just called a "neural network" (NN), is a mathematical model or computational model based on biological neural networks, in other words, is an emulation of biological neural system. It consists of an interconnected group of artificial neurons and processes information using a connectionist approach to computation. In most cases an ANN is an adaptive system that changes its structure based on external or internal information that flows through the network during the learning phase.

\section{Proposed System}

Documentation is the important part of any project. Here in our paper we are doing documentation of projects, which is uploaded by user of the application. Not only we are providing documentation but also one that is user can login at any time and upload his project and can refer the existing documents uploaded by the other user also. Here in our paper we are mainly concentrating on helping students where they can login and make use of the documents to do their project, they can use them as their reference.

\section{IMPLEMENTATION}

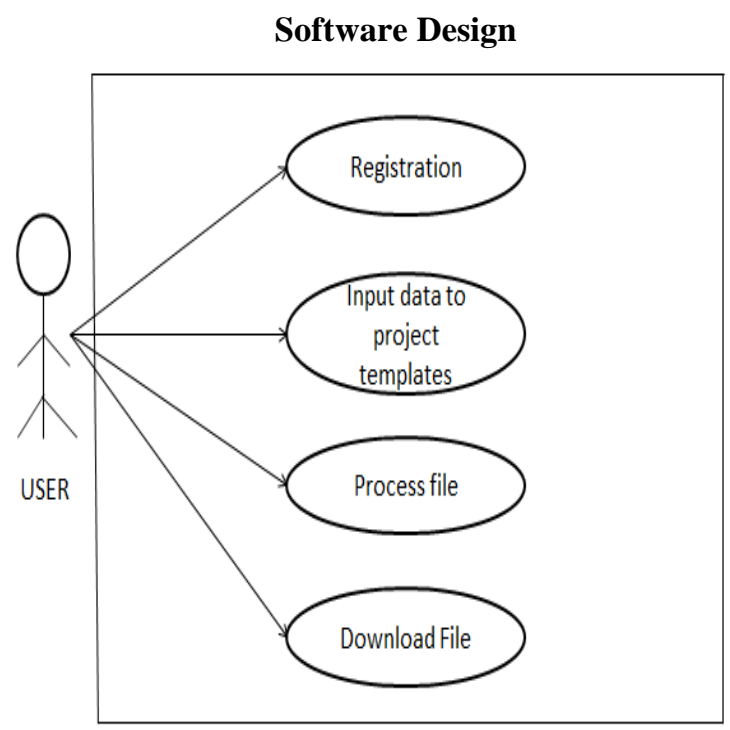

Use case diagram 
Vol. 6, Issue 4, April 2017
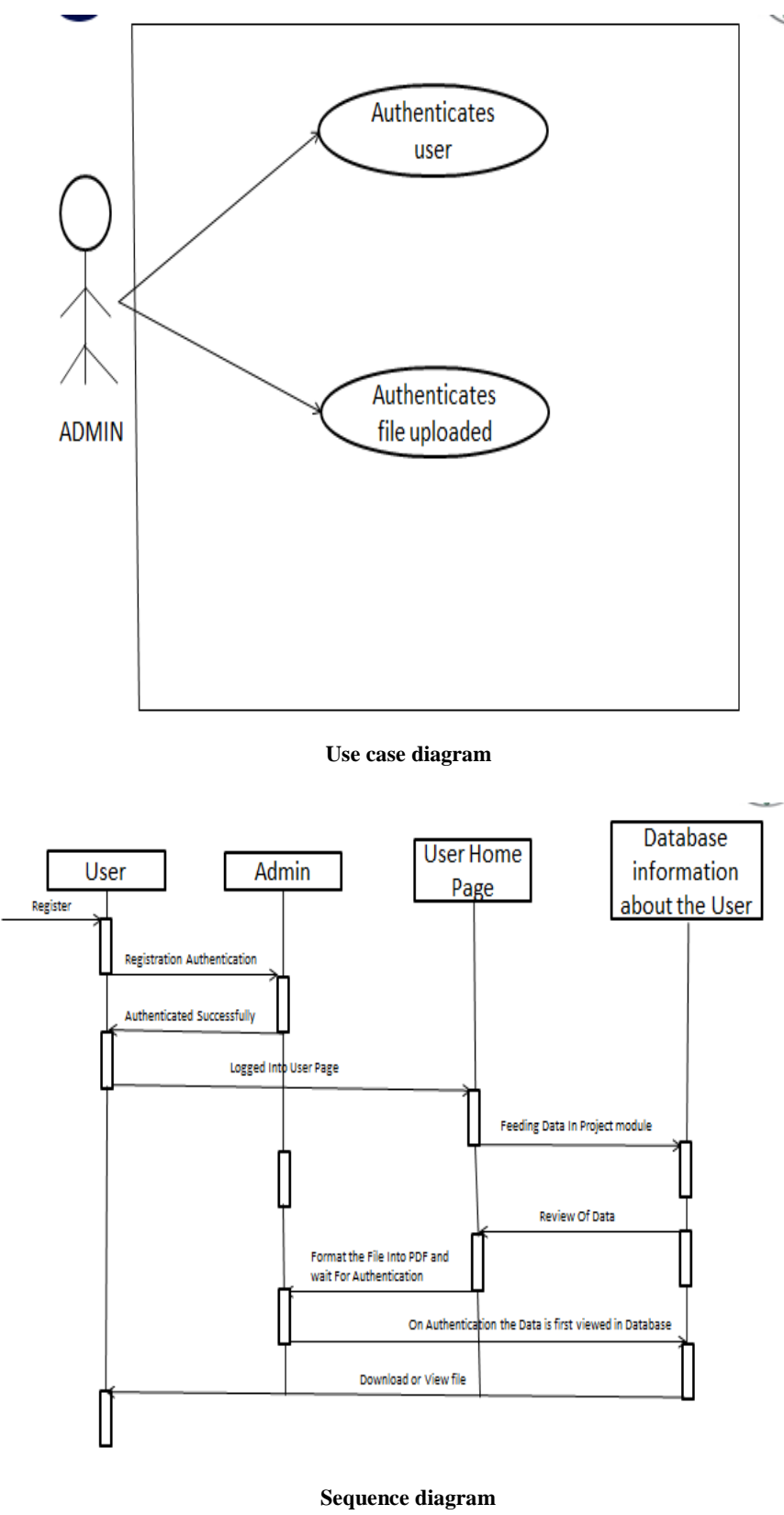

We are using PostgreSQL [4][16] database to store the data and perform various actions on it.

PostgreSQL[17] is a powerful, open source object-relational database system. It has more than 15 years of active development phase and a proven architecture that has earned it a strong reputation for reliability, data integrity, and correctness. PostgreSQL[18][19] (pronounced as post-gress-Q-L) is an open source relational database management system (DBMS) developed by a worldwide team of volunteers. PostgreSQL[20] is not controlled by any corporation or other private entity and the source code is available free of charge.

We make use of Ajax [5] technology to interact with the server through various message transmissions.

Ajax is a client-side script that communicates to and from a server/database without the need for a post back or a complete page refresh. The best definition I've read for Ajax is "the method of exchanging data with a server, and updating parts of a web page - without reloading the entire page." 


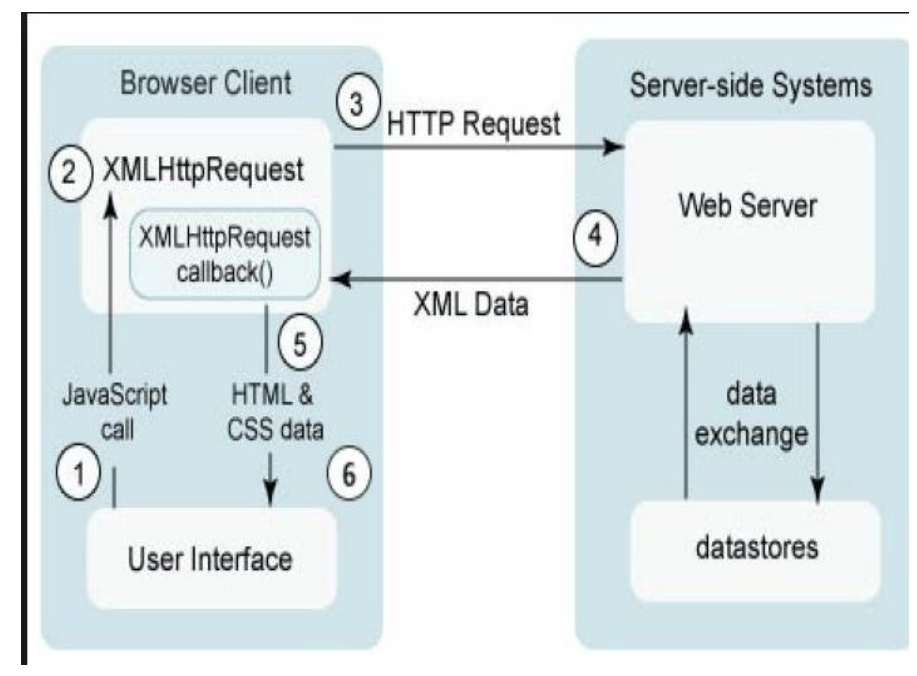

Ajax handling actions

jQuery and JavaScript:

jQuery is a cross-platform JavaScript library designed to simplify the client-side scripting of HTML. It is free, opensource software using the permissive MIT license. Web analysis indicates that it is the most widely deployed JavaScript library by a large margin.

jQuery's syntax is designed to make it easier to navigate a document, select DOM elements, create animations, handle events, and develop Ajax applications. jQuery also provides capabilities for developers to create plug-ins on top of the JavaScript library. This enables developers to create abstractions for low-level interaction and animation, advanced effects and high-level, theme able widgets. The modular approach to the jQuery library allows the creation of powerful dynamic web pages and Web applications.

JavaScript, often abbreviated as "JS", is a high-level, dynamic, untyped, and interpreted run-time language. It has been standardized in the ECMAScript language specification. Alongside HTML and CSS, JavaScript is one of the three core technologies of World Wide Web content production; the majority of websites employ it, and all modern Web browsers support it without the need for plug-ins. JavaScript is prototype-based with first-class functions, making it a multi-paradigm language, supporting object-oriented, imperative, and functional programming styles. It has an API for working with text, arrays, dates and regular expressions, but does not include any I/O, such as networking, storage, or graphics facilities, relying for these upon the host environment in which it is embedded.

\section{XML and HTML:}

In computing, Extensible Markup Language (XML) [5] is a markup language that defines a set of rules for encoding documents in a format that is both human-readable and machine-readable. The W3C's XML[7][13] 1.0 Specification and several other related specifications all of them free open standards define XML[8].

The design goals of XML[9] emphasize simplicity, generality, and usability across the Internet. It is a textual data format with strong support via Unicode for different human languages. Although the design of XML[10][11] focuses on documents, the language is widely used for the representation of arbitrary data structures such as those used in web services.

Hypertext Markup Language (HTML) [6] is the standard markup language for creating web pages and web applications. With Cascading Style Sheets (CSS) and JavaScript it forms a triad of cornerstone technologies for the World Wide Web. Web browsers receive HTML[12] documents from a webserver or from local storage and render them into multimedia web pages. HTMLdescribes the structure of a web page semantically and originally included cues for the appearance of the document.

HTML[14] elements are the building blocks of HTML pages. With HTML[15] constructs, images and other objects, such as interactive forms, may be embedded into the rendered page. It provides a means to create structured documents by denoting structural semantics for text such as headings, paragraphs, lists, links, quotes and other items. 
HTML elements are delineated by tags, written using angle brackets. Tags such as <img> and <image> introduce content into the page directly. Others such as $\langle\mathrm{p}>\ldots</ \mathrm{p}>$ surround and provide information about document text and may include other tags as sub-elements. Browsers do not display the HTML tags, but use them to interpret the content of the page.

\section{Conclusion}

Our project mainly concentrates on helping students and other technical person can also use the app. Initially the documents or project will be uploaded by our team. And hence as the number of users increases the documentation grows. Hence it will be user friendly application where one can upload their documents and refer to other already uploaded documents.

\section{REFERENCES}

[1] CLUSTERING AND CLASSIFICATION: DATA MINING APPROACHES by Ed Colet

[2] A Tutorial on Support Vector Machines for Pattern Recognition CHRISTOPHER J.C. BURGES

[3] Overview of Bayesian network approaches to model geneenvironment interactions and cancer susceptibility Chengwei Su, Angeline Andrew, Margaret Karagas,Mark E. Borsuk.

[4] https://www.tutorialspoint.com/postgresql/postgresql_overview.html

[5] https://en.wikipedia.org/wiki/XML

[6] https://en.wikipedia.org/wiki/HTML

[7] "XML Media Types, RFC 7303". Internet Engineering Task Force. July 2014.

[8] "XML 1.0 Specification". World Wide Web Consortium. Retrieved 22 August 2010.

[9] "XML and Semantic Web W3C Standards Timeline" (PDF). Retrieved 14 August 2016.

[10] "W3C DOCUMENT LICENSE".

[11] "XML 1.0 Origin and Goals". Retrieved 14 August 2016.

[12] Flanagan, David. JavaScript - The definitive guide (6 ed.). p. 1. JavaScript is part of the triad of technologies that all Web developers must learn: HTML to specify the content of web pages, CSS to specify the presentation of web pages, and JavaScript to specify the behaviour of web pages.

[13] "HTML 4.0 Specification W3C Recommendation Conformance: requirements and recommendations". World Wide Web Consortium. December 18, 1997. Retrieved July 6, 2015.

[14] Tim Berners-Lee, "Information Management: A Proposal." CERN (March 1989, May 1990). W3.org

[15] Tim Berners-Lee, "Design Issues

[16] "Debian -- Details of package pgadmin3 in jessie". Retrieved 2017-03-10.

[17] "PostgreSQL licence approved by OSI". Crynwr. 2010-02-18. Retrieved 2010-02-18.

[18] "OSI PostgreSQL Licence". Open Source Initiative. 2010-02-20. Retrieved 2010-02-20.

[19] "What is PostgreSQL?". PostgreSQL 9.3.0 Documentation. PostgreSQL Global Development Group. Retrieved 2013-09-20.

[20] Stonebraker, M; Rowe, LA (May 1986). The design of POSTGRES (PDF). Proc. 1986 ACM SIGMOD Conference on Management of Data. Washington, DC. Retrieved 2011-12-17. 